\title{
LES ENJEUX SOCIAUX DU SOIN PAR LE CONTACT ANIMALIER
}

\author{
Jérôme Michalon
}

Orspere-Samdarra - Observatoire Santé mentale Vulnérabilités et Sociétés | «Rhizome»

2019/2 $\mathrm{N}^{\circ} 72 \mid$ pages 3 à 5

ISSN 1622-2032

Article disponible en ligne à l'adresse :

https://www.cairn.inforevue-rhizome-2019-2-page-3.htm

Distribution électronique Cairn.info pour Orspere-Samdarra - Observatoire Santé mentale Vulnérabilités et Sociétés.

(C) Orspere-Samdarra - Observatoire Santé mentale Vulnérabilités et Sociétés. Tous droits réservés pour tous pays.

La reproduction ou représentation de cet article, notamment par photocopie, n'est autorisée que dans les limites des conditions générales d'utilisation du site ou, le cas échéant, des conditions générales de la licence souscrite par votre établissement. Toute autre reproduction ou représentation, en tout ou partie, sous quelque forme et de quelque manière que ce soit, est interdite sauf accord préalable et écrit de l'éditeur, en dehors des cas prévus par la législation en vigueur en France. Il est précisé que son stockage dans une base de données est également interdit. 


\section{Jérôme Michalon}

Chargé de recherche en sociologie

CNRS

UMR Triangle - Université Jean-Monnet

Saint-Étienne

\section{Les enjeux sociaux du soin par le contact animalier}



Un cheval nommé Peyo a récemment fait parler de lui dans les médias et sur les réseaux sociaux. En effet, cet imposant équidé rend visite deux fois par mois aux résidents d'un établissement d'hébergement pour personnes âgées dépendantes (Ehpad) dijonnais. Ce qui permet à Peyo, cet étalon de 500 kilos, de passer la porte de l'établissement? Il fait " du bien » aux pensionnaires, au point d'être même parfois qualifié de "thérapeute ". Pourtant, Peyo n'est pas le premier animal à se faire une place dans l'univers médicosocial. Depuis plusieurs années, en effet, certaines pratiques de soin, paramédicales, ou d'accompagnement social, mobilisent la présence ou le contact d'animaux, dont on utilise les qualités de " médiateur ", de " catalyseur émotionnel ", de "soutien psychologique ".

Plusieurs terminologies existent pour désigner ces pratiques : " zoothérapie ", " médiation animale ", " équithérapie ", " delphinothérapie ", etc. Dans la thèse que j'ai consacrée à l'émergence de ces pratiques ${ }^{1}$, j'ai utilisé le terme "soin par le contact animalier " qui me semblait rendre bien compte de tout ce que ces pratiques avaient en commun. Toutes procèdent en effet de la mise en relation intentionnelle, par un tiers, d'un animal vivant et d'un humain en souffrance et/ou en situation de handicap, dans le but de produire un bénéfice chez ce dernier. Une fois cette définition large posée, il est possible d'évoquer l'histoire du développement de ces activités peu communes, témoignant de la rencontre entre prise en charge de la santé humaine et souci de l'animal. Ces pratiques émergent à partir des années 1960, en Europe de l'Ouest et dans le monde anglophone, conjointement ou presque aux recherches qui viendront leur donner une assise scientifique (peu assurée malgré tout encore aujourd'hui). C'est autour de deux espèces particulières, le chien et le cheval, que cette histoire s'est écrite et elle témoigne des changements conséquents de nos rapports à ces animaux.

\section{Revaloriser la compagnie du chien}

Depuis le $x X^{e}$ siècle, le chien domestique a connu une évolution importante dans les sociétés occidentales : il est devenu progressivement la figure paradigmatique de "I'animal de compagnie ". De multiples définitions seraient à proposer à propos de ce statut, mais on peut retenir parmi celles-ci le fait qu'un animal de compagnie est un animal soustrait à l'exigence de travail et d'utilité. C'est précisément cet élément qui est l'objet des critiques régulièrement adressées aux propriétaires d'animaux de compagnie : le fait qu'ils dépensent énormément d'argent pour le soin d'animaux n'ayant aucune autre utilité que l'agrément.

Pour comprendre l'émergence du soin par le contact animalier, il faut avoir en tête l'existence de ces critiques et la force sociale de cette obligation d'utilité faite à l'animal domestique. À ce titre, avec l'inclusion des chiens dans les pratiques de soin, nous assistons à la création d'une nouvelle forme d'utilité sociale pour l'espèce. Cette inclusion s'est faite par étapes : au début $d u x^{\mathrm{e}}$ siècle, se développent les chiens-guides d'aveugle, puis dans les années 1960, les chiens écouteurs pour déficients auditifs et les chiens d'assistance pour personnes en fauteuil roulant, au milieu des années 1970 . On peut qualifier ces pratiques comme relevant de l'assistance animalière, puisqu'il s'agit de former un animal à l'exécution de certaines tâches (guider et avertir essentiellement) censées aider la personne handicapée à évoluer dans son environnement quotidien. S'inspirant pour partie des pratiques d'assistance, les pratiques de soin par le contact canin, à proprement parler, se développent dans les années 1970, dans un contexte où le marché de l'animal de compagnie prend un essor considérable. Les industriels de ce secteur (les fabricants de croquettes, au premier chef) seront très intéressés par le développement de ces pratiques qui viennent apporter un contrepoint parfait aux critiques adressées aux propriétaires d'animaux de compagnie, évoquées plus haut. 
Cette nouvelle forme d'utilité pour les chiens est l'illustration parfaite qu'une relation bienveillante, affectueuse, insistant sur la singularité des animaux, n'est pas nécessairement synonyme de repli sur soi, de narcissisme et génératrice de sentiments misanthropes. Au contraire, cette relation peut être mise au service d'objectifs altruistes, humanistes. En un mot : avec le soin par le contact canin, l'amour des animaux peut être bénéfique à tous. Les vétérinaires pour animaux de compagnie ainsi que les associations de protection animale ne pouvant que partager un tel credo vont, avec les industriels des animaux de compagnie, investir de l'argent et de l'énergie dans la promotion et la structuration des pratiques de soin par le contact canin. C'est la formation de ce que j'ai appelé la communauté Human-animal interactions (HAI). Dans un premier temps, cette communauté va encourager et financer les recherches sur les interactions avec I'animal à but thérapeutique, en prenant acte du peu de savoirs scientifiques existant sur la question.

À partir du début des années 1980, cette recherche va donc se développer en s'attachant à prouver les bénéfices sanitaires du contact animalier, selon le modèle de recherche le plus légitime dans le monde médical : celui de la médecine de la preuve. L'adoption de ce modèle va faire évoluer le périmètre initial du soin par le contact canin. Les recherches pionnières, à l'image de celles de Boris Levinson ou des époux Corson ${ }^{3}$, étaient clairement du côté du soin psychique, et rendaient compte d'études de cas cliniques. Pour des raisons relevant tout autant de la réflexion stratégique que d'un " sens commun de la science " peu réflexif, les recherches qui suivront vont dénigrer les études de cas et adopter le modèle de l'evidence-based medecine (EMB) pour évaluer les bénéfices physiologiques du contact animalier. Les vertus apaisantes, relaxantes, du contact avec certains animaux sont aujourd'hui bien documentées, de même que les bénéfices sur la santé cardiovasculaire. En revanche, les recherches " butent " bien souvent sur ce qui explique la survenue de ces bénéfices : I'animal en lui-même ou le type de relation à l'animal. Cela s'explique par l'adoption du modèle de la médecine de la preuve qui, élaboré pour évaluer des traitements médicamenteux, considère la dimension relationnelle (c'est-à-dire la représentation que le patient se fait de son traitement) comme un obstacle, un biais à éliminer au maximum, si l'on veut prouver l'efficacité d'un traitement. La situation dans laquelle s'est trouvée, et se trouve encore, la recherche autour des interactions avec l'animal à but thérapeutique est la suivante : évaluer l'efficacité d'une pratique psychothérapeutique (puisqu'initialement, il s'agit de cela) avec des outils forgés pour mettre à distance la dimension relationnelle, psychique et sociale, présente dans tout travail de soin.

\section{À la recherche du label « thérapeutique »}

2 Levinson, B. M. (1962). The dog as "co-therapist". Mental Hygiene, $46,59-65$.

$\underline{3}$ Corson, S. A., Corson, E. O., et al. (1975). Pet-facilitated psychotherapy in a hospital setting. Dans J. H. Masserman, Current Psychiatric Therapies (p. 277-286). New York: Grune and Stratton.
Pourquoi s'être engagé dans ce qui paraît être une impasse? Tout simplement pour les raisons évoquées plus haut, à savoir : la nécessité de prouver "scientifiquement " que le contact avec l'animal est bénéfique pour la santé humaine, pour servir au mieux les objectifs de la communauté HAI (soit promouvoir des rapports respectueux aux animaux et légitimer un marché de l'animal de compagnie socialement stigmatisé). Le souci de professionnalisation explique également cette direction paradoxale prise par les recherches. Partant du constat du développement hiératique du soin par le contact canin, la communauté HAI défend l'idée que c'est par la validation scientifique (au sens de I'EBM) que les praticiens vont pouvoir se faire accepter du monde médical. II est vrai que l'apparition de " thérapies assistées par l'animal " pratiquées par des personnes n'ayant aucune formation de soignant, et encore moins de thérapeutes, a eu de quoi irriter les professionnels patentés. C'est du côté des professions paramédicales que la réaction a été la plus vive, celles-ci ayant dû lutter pour obtenir le statut de " thérapies " par les autorités médicales.

C'est pour mettre fin à ces débats qu'une terminologie particulière est proposée par la Delta Society - un organisme de formation et de promotion du soin par le contact animalier qui a été créé par la communauté $\mathrm{HAl}$ - : la différence doit être faite entre des " activités associant l'animal (AAA) " (animal-assisted activities) et des " thérapies assistées par l'animal (TAA) " (animal-assisted therapy). Les premières peuvent être réalisées par tout un chacun, ou presque, qui voudrait venir avec son chien dans une maison de retraite pour distraire les résidents, par exemple. Elles n'ont aucune visée thérapeutique ou soignante, donc. Les secondes, en revanche, impliquent d'être pratiquées par des thérapeutes reconnus comme tels par leurs pairs. Psychothérapeute, psychiatre, infirmière, kinésithérapeute, psychomotricien, orthophoniste... : tout professionnel du médical ou du paramédical souhaitant intégrer le contact animalier dans sa pratique peut, sous réserve d'une formation particulière, se revendiquer de la TAA.

L'innovation terminologique n'est pas déconnectée des enjeux de structuration professionnelle et du respect des juridictions déjà en place dans le monde médical. Elle traduit l'adoption de ce que j'ai nommé la politique de la " thérapie aux thérapeutes ». Initiée dans les années 1990, d'abord aux États-Unis, cette politique a été adoptée partout où les pratiques de soin par le contact animalier ont émergé. En France, elle est apparue explicitement au début des années 2000 - moment où l'on a affirmé explicitement la différence entre AAA et TAA - et elle a progressivement évolué vers une intégration de professionnels non issus du monde médical, comme les travailleurs sociaux. Cette évolution a débouché sur l'apparition, en 2005, du terme de " médiation animale ", qui à la fois témoignait de cette volonté d'ouverture plus large à ces professionnels non médicaux, mais aussi d'une envie de ne plus utiliser le terme de "thérapie " (du fait des tensions vives qu'il pouvait susciter vis-à-vis du monde médical) et aussi de se rapprocher d'autres pratiques de " médiation " (art thérapie, thérapie corporelle, musico thérapie).

S'il est donc moins question de thérapie, le consensus, toutefois, reste le même : pour pouvoir être professionnel de la médiation animale, il faut d'abord être professionnel du soin et/ou de l'accompagnement des humains. Ce qui explique que toutes les formations existantes en médiation animale, en France comme ailleurs, insistent sur l'idée de former à une "spécialisation " et non pas à une profession à part entière. 


\section{Faire du cheval "plus qu'une monture »}

II a beaucoup été question du chien dans ce qui vient d'être évoqué. Pourtant, le cheval est tout autant présent dans le soin par le contact animalier. Le soin par contact équin s'est développé à peu près à la même période que celles engageant les chiens, voire un peu antérieurement. Dans les années 1960, une forme d'équitation adaptée aux personnes présentant des handicaps physiques et moteurs fait son apparition en Europe puis en Amérique du Nord. Constatant les bienfaits de cette activité sur les capacités motrices, des kinésithérapeutes ont mis en place de la rééducation par l'équitation, qui signa l'entrée du cheval dans le soin à part entière. À la fin des années 1970, dans le sillage de l'émergence de la psychomotricité, une thérapie s'orientant plus sur les dimensions psychiques fait son apparition (sous le nom de « thérapie avec le cheval " en France et d'" hippotherapy " aux États-Unis). Une rupture s'exprime alors : il n'est plus question d'équitation ici, de son utilisation ou de son apprentissage, mais du rapport au cheval, comme animal " sensible ", doté d'un psychisme particulier, qui le rend intéressant dans le cadre d'un travail thérapeutique. Cette vision du cheval sera d'ailleurs au cœur du développement dans les années 2000, de nouvelles pratiques, comme l'équithérapie en France ou l'Equine facilitated psycotherapy aux États-Unis, qui revendiqueront une orientation clairement psychothérapeutique. L'histoire du soin par le contact équin est celle d'une prise de distance vis-à-vis du monde de l'équitation, de la revendication d'un nouveau statut pour le cheval, vu autrement que comme une monture. En cela, cette histoire fait écho aux évolutions plus globales des rapports aux chevaux.

Rappelons en effet que depuis le début du xxe siècle, le cheval a perdu progressivement une partie de ses fonctions productives (avec la mécanisation des moyens de transport), pour devenir majoritairement un animal consacré aux loisirs (jeux hippiques et équitation sportive et de loisir). Son statut social a évolué en direction d'une plus grande prise en compte de son individualité, de sa sensibilité, dont témoignent l'apparition depuis une trentaine d'années de nouvelles pratiques d'équitation (monte éthologique, chuchoteurs), voire de rapports aux chevaux en dehors du cadre de l'équitation (par exemple, les " équipiétons ", ces propriétaires de chevaux qui ne les montent pas).
Le cheval, " animal intermédiaire ${ }^{4}$ » dont le statut se situe entre celui d'animal de rente et celui d'animal de compagnie, semble de plus en plus proche de ce second pôle.

L'assimilation du cheval à l'animal de compagnie est donc une tendance forte, dans laquelle l'apparition du soin par le contact équin joue un rôle important me semble-t-il. C'est clairement la revendication du statut de thérapeute, professionnel du monde médical qui permet aux praticiens de se détacher du monde de l'équitation ainsi que de défendre une nouvelle vision des équidés et, par-là, une nouvelle utilité pour le cheval, qui prendrait en compte sa sensibilité, son individualité, voire sa personnalité (le terme revient souvent). La dynamique de rapprochement avec les chiens (" de compagnie » et de " soin ») est ici saillante. Pourtant, on ne peut que constater que le soin par le contact canin ainsi que le soin par le contact équin se sont développés de manière parallèle, hormis quelques échanges sporadiques, et constituent encore deux mondes sociaux isolés. Notons d'ailleurs que du côté cheval, on utilise des terminologies qui mentionnent l'espèce (" équithérapie ", " hippotherapy ", thérapie avec le cheval), alors que les terminologies généralistes (" thérapie assistée par l'animal », " zoothérapie » ou « médiation animale ») proviennent d'acteurs plutôt en lien avec le monde du chienํ․

\section{Soigner par le soin}

Ces terminologies généralistes ne disent donc rien des animaux mobilisés dans le soin par le contact animalier, et encore moins du type de relation à l'animal. Or, ce n'est pas n'importe quel rapport à l'animal qui se donne à voir lorsque l'on observe les pratiques. D'une part, c'est un rapport individualisé : I'animal n'est jamais considéré comme un simple exemplaire de son espèce, et on insiste sur son caractère, son individualité, ses humeurs, etc. En somme, on met en avant tout ce qui distingue tel animal de ses congénères. Ces qualités individuelles sont censées faire écho à l'individualité des bénéficiaires, patients, usagers, humains. La relation de soin qui s'établit alors est thématisée par les professionnels comme celle d'une rencontre entre deux individualités, deux personnalités. Rapports personnalisés donc, mais également rapports bienveillants aux animaux. Tout au long des séances de soin par le contact animalier, il est constamment rappelé aux bénéficiaires que les animaux doivent être traités avec égards, respect, bienveillance. II est par ailleurs frappant d'observer que le soin aux animaux (les nourrir, les nettoyer, les caresser, leur témoigner de la sollicitude, de l'affection) est omniprésent dans les séances. Ces soins ne sont jamais optionnels et occupent parfois toute la séance. Ces moments sont centraux, selon les thérapeutes, pour travailler avec les bénéficiaires sur différentes dimensions de la vie psychique, émotionnelle et même sur la motricité fine (tenir une brosse et savoir s'en servir n'est pas à la portée de tout le monde). Ici, le soin aux animaux est intimement lié au soin des humains. Même : c'est en soignant les animaux que les humains se soignent.

C'est dans cette mesure qu'il me semble adapté de parler de " soin par le contact animalier " car, à défaut de préciser de quels animaux il est question, l'expression indique plus clairement quel type de relation est à l'œuvre dans ces pratiques : le soin des humains et le soin des animaux. Le soin comme régime relationnel qui place en son centre l'intrication entre la prise en compte de l'individualité, de la singularité des êtres et l'actualisation d'une bienveillance à leur égard. D 